\title{
Power bounded composition operators on spaces of analytic functions
}

\author{
José Bonet · Paweł Domański
}

Received: 15 February 2010 / Accepted: 21 June 2010 / Published online: 20 October 2010

(C) The Author(s) 2010. This article is published with open access at Springerlink.com

\begin{abstract}
We study the dynamical behaviour of composition operators defined on spaces of real analytic functions. We characterize when such operators are power bounded, i.e. when the orbits of all the elements are bounded. In this case this condition is equivalent to the composition operator being mean ergodic. In particular, we show that the composition operator is power bounded on the space of real analytic functions on $\Omega$ if and only if there is a basis of complex neighbourhoods $U$ of $\Omega$ such that the operator is an endomorphism on the space of holomorphic functions on each $U$.
\end{abstract}

Keywords Spaces of real analytic functions - Real analytic manifold - Composition operator · Mean ergodic operator · Power bounded operator · Orbit · Hypercyclic operator . Hyperbolic spaces

Mathematics Subject Classification (2000) Primary 47B33 - 46E10; Secondary $47 \mathrm{~A} 16 \cdot 47 \mathrm{~A} 35$

\section{Introduction and notation}

The purpose of this paper is to study the behaviour of orbits of composition operators $C_{\varphi}(f):=f \circ \varphi, \varphi$ a real analytic self map, on spaces of real analytic functions defined

Dedicated to the memory of our friend Klaus D. Bierstedt.

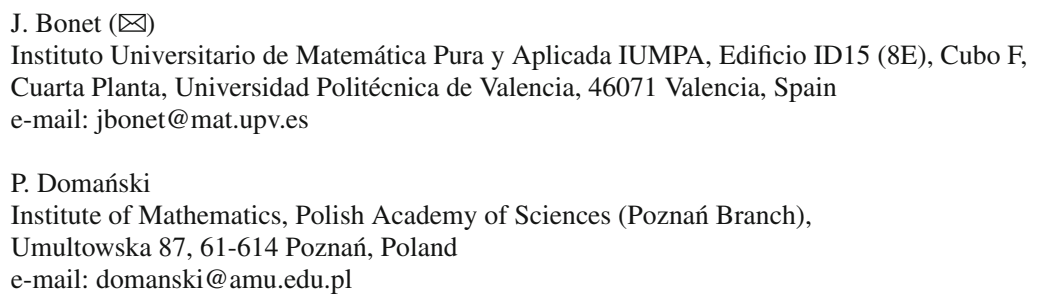


on an open subset of $\mathbb{R}^{d}$ or, more generally, on a real analytic manifold. There are three interesting notions describing different dynamical behaviour of a continuous linear operator: power boundedness (i.e. all orbits are bounded), mean ergodicity (i.e. all orbits are Cesaro convergent, see $[29,41])$ and hypercyclicity (i.e. there exists a dense orbit). We completely characterize when $C_{\varphi}$ is power bounded in terms of the self map $\varphi$. In particular we prove that in this context $C_{\varphi}$ is power bounded if and only if it is mean ergodic. Our results provide a new, rather surprising, necessary condition for a composition operator to be hypercyclic.

A systematic investigation of composition operators on spaces of real analytic functions have been undertaken by Langenbruch and the second author; see the series of papers [15-17]. However, these papers concentrate on aspects different form the dynamical behaviour of the operator.

There is a huge literature about the dynamical behavior of various linear continuous operators on Banach, Fréchet and more general locally convex spaces; see the survey paper by Grosse-Erdmann [21] and the recent books by Bayart and Matheron [4] and by GrosseErdmann and Peris [23]. Composition operators on different function spaces have been also extensively investigated. See, for instance, [5,6,22,38]; for general theory of composition operators on Banach spaces of holomorphic functions see [11,37].

The space $\mathscr{A}(\Omega)$ of real analytic functions, i.e. analytic complex functions with real argument in $\Omega \subseteq \mathbb{R}^{d}$ or $\Omega$ being a real analytic manifold, is a natural function space, which has attracted much attention recently in connection with its topological structure (for example the space is separable, complete and has no Schauder basis [18]), global analysis, the splitting of short exact sequences, composition operators, and surjectivity, existence of continuous linear right inverse and parameter dependence of linear partial differential operators. See, e.g. [30,31,33,39], the authors' papers [7,8,13,15-17] and the survey [12].

Let $T: E \rightarrow E$ be a continuous linear operator on a Hausdorff locally convex space $E$. The iterates of $T$ are denoted by $T^{n}:=T \circ \cdots \circ T, n \in \mathbb{N}$. If the sequence $\left(T^{n}\right)_{n \in \mathbb{N}}$ is equicontinuous in the space $L(E)$ of linear operators from $E$ to $E, T$ is called power bounded. In case the space $E$ is barrelled, for example if $E=\mathscr{A}(\Omega)$, one can apply the uniform boundedness principle to conclude that $T$ is power bounded if and only if the orbit $\left\{T^{n}(x): n \in \mathbb{N}\right\}$ is bounded for every $x \in E$.

A continuous linear operator $T$ on $E$ is called mean ergodic if the limits

$$
P x:=\lim _{N \rightarrow \infty} \frac{1}{N} \sum_{n=1}^{N} T^{n} x, \quad x \in E,
$$

exist in $E$. A power bounded operator $T$ is mean ergodic precisely when $X=\operatorname{ker}(I-T) \oplus$ $\overline{\operatorname{im}(I-T)}$. Moreover, $\operatorname{im} P=\operatorname{ker}(I-T)$ and $\operatorname{ker} P=\overline{\operatorname{im}(I-T)}$. If the space $E$ is barrelled and $T$ is mean ergodic, the sequence $\frac{1}{N} \sum_{n=1}^{N} T^{n}$ converges pointwisely to a continuous linear projection $P$; see [41, Ch. VIII, Sect. 3]. If the convergence is uniform on bounded sets we call $T$ uniformly mean ergodic. There is a classical theory of mean ergodic operators which goes back to fundamental papers of Yosida and Hille especially in the Banach case; cf. [20] and [29]. For more details on the locally convex theory see [2,3,41] and the references therein.

Let $\varphi: U \rightarrow U$ be a continuous self-map on a topological space $U$, we say that $\varphi$ has stable orbits on $U$ if for every compact subset $K$ of $U$ there is a compact subset $L \Subset U$ such that $\varphi^{n}(K) \subseteq L$ for $n \in \mathbb{N}$. This concept plays an important role in the characterizations below. 
Main Theorem Let $\Omega$ be a real analytic manifold (compact or non-compact) and let $\varphi$ : $\Omega \rightarrow \Omega$ be a real analytic map. The following assertions are equivalent:

(a) $C_{\varphi}: \mathscr{A}(\Omega) \rightarrow \mathscr{A}(\Omega)$ is power bounded.

(b) $C_{\varphi}: \mathscr{A}(\Omega) \rightarrow \mathscr{A}(\Omega)$ is (uniformly) mean ergodic.

(c) The manifold $\Omega$ has a basis of complex neighbourhoods consisting of sets $V$ such that $\varphi$ extends as a holomorphic self-map of $V$.

(d) There is a complex neighbourhood $V$ of $\Omega$ such that $\varphi$ extends to $V$ as a holomorphic self map and $\varphi$ has stable orbits on $V$, or equivalently $C_{\varphi}: H(V) \rightarrow H(V)$ is power bounded; see [9].

In particular, if $C_{\varphi}: \mathscr{A}(\Omega) \rightarrow \mathscr{A}(\Omega)$ is hypercyclic, then (c) does not hold.

In Corollary 2.6 we get a precise description of $\varphi$ such that $C_{\varphi}: \mathscr{A}(\Omega) \rightarrow \mathscr{A}(\Omega)$ is power bounded, which gives a very strong necessary condition. A neat description is obtained for real analytic functions on intervals of the real line in Theorem 2.8.

A description of the natural topology on $\mathscr{A}(\Omega)$ (going back to Martineau) is given, for instance, in [12] or [18]. The space $\mathscr{A}(\Omega)$ has very good properties: it is nuclear, separable, complete, barrelled and even ultrabornological, satisfies the closed graph theorem, but surprisingly it has no Schauder basis by [18].

To be precise, the space $\mathscr{A}(\Omega)$ is equipped with the unique locally convex topology such that for any $U \subseteq \mathbb{C}^{d}$ open, $\mathbb{R}^{d} \cap U=\Omega$, the restriction map $R: H(U) \longrightarrow \mathscr{A}(\Omega)$ is continuous and for any compact set $K \subseteq \Omega$ the restriction map $r: \mathscr{A}(\Omega) \longrightarrow H(K)$ is continuous. We endow the space $H(U)$ of holomorphic functions on $U$ with the compactopen topology and the space $H(K)$ of germs of holomorphic functions on $K$ with its natural locally convex inductive limit topology:

$$
H(K)=\operatorname{ind}_{n \in \mathbb{N}} H^{\infty}\left(U_{n}\right),
$$

where $\left(U_{n}\right)_{n \in \mathbb{N}}$ is a basis of $\mathbb{C}^{d}$-neighbourhoods of $K$. Martineau (see [12]) proved that there is exactly one topology on $\mathscr{A}(\Omega)$ satisfying the condition above. Endowed with this topology one has the following description as a countable projective limit:

$$
\mathscr{A}(\Omega)=\operatorname{proj}_{N \in \mathbb{N}} H\left(K_{N}\right) .
$$

Here $\left(K_{N}\right)_{N}$ is a fundamental sequence of compact subsets of $\Omega$. Analogously one defines the topology on $\mathscr{A}(\Omega)$ when $\Omega$ is a real analytic manifold [40]. A long survey on the space of real analytic functions with very precise description of its topology is contained in [14].

It is known that every real analytic manifold $\Omega$ has a complexification $\tilde{\Omega}$ [24, Th. III.3.3] and it has a fundamental system of Stein open neighbourhoods in $\tilde{\Omega}$ [24, Th. III.3.6]. Clearly, if $\Omega \subseteq \mathbb{R}^{d}$ is just an open subset then $\tilde{\Omega}$ is an open subset of $\mathbb{C}^{d}$ and a complex neighbourhood of $\Omega$. By a complex neighbourhood of a subset $S$ of a real analytic manifold $\Omega$ we mean a neighbourhood of $S$ in a complexification $\tilde{\Omega}$ of $\Omega$. Again in case of open sets $\Omega \subseteq \mathbb{R}^{d}$ a complex neighbourhood of $S \subseteq \Omega$ is just a neighbourhood of $S$ in $\mathbb{C}^{d}$. Moreover, without loss of generality we may assume that the complexification $\tilde{\Omega}$ of $\Omega$ is embedded as a Stein manifold into some $\mathbb{C}^{d}$ such that $\tilde{\Omega} \cap \mathbb{R}^{d}=\Omega$ see [24, Th. VI.1.1]. Recall that every Stein manifold embeds via a proper map into $\mathbb{C}^{d}$ for suitable $d \in \mathbb{N}[26,5.3 .9]$.

We will use Kobayashi semi-distance $k_{V}(\cdot, \cdot)$ on a complex manifold $V \subseteq \mathbb{C}^{d}$. Here the beautiful book [27] is a standard reference. Every holomorphic map $\varphi: V \rightarrow V$ is always non-expansive with respect to $k_{V}$. The manifold $V$ is called hyperbolic if $k_{V}$ is a distance (and then it induces the standard topology of $V$ ). Every domain biholomorphic to a bounded set is automatically hyperbolic [27, Cor. 4.1.10, Prop. 3.2.2]. The manifold $V$ is called Kobayashi 
complete if $\left(V, k_{V}\right)$ is a complete metric space, or equivalently, if every ball in this space is relatively compact [27, Prop. 1.1.9]. Every bounded open set $V \subseteq \mathbb{C}^{d}$ such that every its boundary point admits a weak peak function is Kobayashi complete [27, Cor. 4.1.11]. Both hyperbolicity and Kobayashi completeness are biholomorphic invariants.

By $B(x, r)$ and $B_{k_{V}}(x, r)$ we denote, respectively, euclidean and Kobayashi balls of center $x$ and radius $r$. The notation $K \Subset U$ means that $K$ is a compact subset of the open set $U$. By $\overline{\mathbb{R}}$ we denote the extended real line $\mathbb{R} \cup\{\infty\} \cup\{-\infty\}$. For non-explained notions from functional analysis we refer to [35]. For complex analysis of several variables see [25] and for real analytic manifolds see [24]. For dynamics of holomorphic maps see [1,36].

\section{Proof of the main theorem}

Theorem 2.1 Let $\Omega$ be a real analytic manifold (compact or non-compact) and let $\varphi: \Omega \rightarrow$ $\Omega$ be a real analytic map. The following assertions are equivalent:

(a) $C_{\varphi}: \mathscr{A}(\Omega) \rightarrow \mathscr{A}(\Omega)$ is power bounded.

(b) $C_{\varphi}: \mathscr{A}(\Omega) \rightarrow \mathscr{A}(\Omega)$ is uniformly mean ergodic.

(c) $C_{\varphi}: \mathscr{A}(\Omega) \rightarrow \mathscr{A}(\Omega)$ is mean ergodic.

(d) For all $K \Subset \Omega$ there is $L \Subset \Omega$ such that for every complex neighbourhood $U$ of $L$ there is a complex neighbourhood $V$ of $K$ such that

$$
\forall n \in \mathbb{N} \quad \varphi^{n} \text { is defined on } V \text { and } \varphi^{n}(V) \subseteq U .
$$

(e) For every complex neighbourhood $U$ of $\Omega$ there is a complex (open!) neighbourhood $V \subseteq U$ of $\Omega$ such that $\varphi$ extends as a holomorphic function to $V, \varphi(V) \subseteq V$, and $\varphi$ has stable orbits on $V$.

(f) The manifold $\Omega$ has a basis of complex neighbourhoods consisting of sets $V$ such that $\varphi$ extends as a holomorphic self-map of $V$.

Proof Recall that the manifold $\Omega$ and its complexification $\tilde{\Omega}$ are embedded as submanifolds into $\mathbb{C}^{d}$ such that $\tilde{\Omega} \cap \mathbb{R}^{d}=\Omega$.

(a) $\Rightarrow$ (b): Follows from [2, Prop. 2.4] since $\mathscr{A}(\Omega)$ is Montel and all its bounded subsets are metrizable; see also [10].

(b) $\Rightarrow$ (c): Obvious.

(c) $\Rightarrow$ (d): By [2, Prop. 2.2], the sequence $\left(\frac{1}{n} C_{\varphi^{n}}\right)$ tends to zero, so it is bounded and equicontinuous on $\mathscr{A}(\Omega)$, since $\mathscr{A}(\Omega)$ is barrelled. Now, it is a standard argument that for every $K \Subset \Omega$ there is $L \Subset \Omega$ such that each $C_{\varphi^{n}}$ acts continuously from $\mathscr{A}(\Omega)$ equipped with the topology of $H(L)$ into $\mathscr{A}(\Omega)$ equipped with the topology of $H(K)$ and, moreover, $\left(\frac{1}{n} C_{\varphi^{n}}\right)$ is an equicontinuous family with respect to these topologies. Since $\mathscr{A}(\Omega)$ is a dense subspace of the complete spaces $H(K)$ and $H(L)$, each $C_{\varphi^{n}}$ extends to continuous operators $\widehat{C_{\varphi^{n}}}: H(L) \longrightarrow H(K)$ and $\left(\frac{1}{n} \widehat{C_{\varphi^{n}}}\right)$ is an equicontinuous family. Let us emphasize that $\widehat{C_{\varphi^{n}}}$ is just an extension via density so, a priori, there is no reason why it can be defined as a composition operator on $H(L)$.

We first show that, for every $n \in \mathbb{N}$,

$$
\varphi^{n}(K) \subseteq L
$$

Of course, if $\Omega$ is compact we can take $K=L=\Omega$. In the general case assume that $\varphi^{n}(K) \nsubseteq L$, thus there is $u=\left(u_{1}, \ldots, u_{d}\right) \in \varphi^{n}(K)$ such that $u \notin L$. Let 


$$
f_{\varepsilon}(z):=\frac{1}{\sum_{j=1}^{d}\left(z_{j}-u_{j}\right)^{2}+\varepsilon i} .
$$

Clearly for every $\varepsilon>0$ the function $f_{\varepsilon} \in \mathscr{A}(\Omega)$. As $\varepsilon \rightarrow 0, f_{\varepsilon}(z) \rightarrow \frac{1}{\sum_{j=1}^{d}\left(z_{j}-u_{j}\right)^{2}} \in H(L)$ on a complex neighbourhood of $L$ but $\left|f_{\varepsilon} \circ \varphi^{n}(v)\right| \rightarrow \infty$ for any $v \in K, \varphi^{n}(v)=u$; a contradiction.

Let $U$ be a complex neighbourhood of $L$. We may assume without loss of generality that $U$ is a polynomial polyhedron, or more precisely an intersection of a polynomial polyhedron in $\mathbb{C}^{d}$ with $\tilde{\Omega}$ see [26, Lemma 2.7.4], comp. [19, Proof of Th. 1.6]. Since $\frac{1}{n} \widehat{C_{\varphi^{n}}}: H(L) \longrightarrow$ $H(K)$ are equicontinuous, for every $U_{1} \Subset U$ a complex neighbourhood of $L$ there is $V$ a complex neighbourhood of $K$ such that

$$
\widehat{C_{\varphi^{n}}}: H^{\infty}\left(U_{1}\right) \longrightarrow H^{\infty}(V)
$$

is continuous for every $n \in \mathbb{N}$. The reason for that is that any equicontinuous family of operators between LB-spaces maps a fixed step-space into some fixed step-space continuously.

We consider $U \subseteq \tilde{U}$ such that $\tilde{U}$ is a complex neighbourhood of $\Omega$ such that $\varphi: \tilde{U} \longrightarrow \mathbb{C}^{d}$ is defined and holomorphic and, without loss of generality, we may assume that $V \subset \tilde{U}$. Since $\varphi(K) \subseteq L$, we may also assume without loss of generality that $\varphi(V) \subseteq U$. Now, assume that $n$ is the smallest natural number $k$ such that the following does not hold:

$$
\varphi^{k} \text { is defined on } V \text { and } \varphi^{k}(V) \subseteq U .
$$

We have chosen $U, V$ in such a way that $n>1$. Since $\varphi^{n-1}$ is defined on $V$ and $\varphi^{n-1}(V) \subseteq$ $U \subseteq \tilde{U}$ so $\varphi^{n}$ is defined on $V$. Therefore $\varphi^{n}(V) \nsubseteq U$.

Let $u \in \varphi^{n}(V) \backslash U$. Since $U$ is assumed to be a polynomial polyhedron, there is a nonconstant polynomial $p$ such that for every $w \in U,|p(w)|<1$ but $p(u) \geq 1$. On the other hand, there is $0<\delta<1$ such that $|p(w)|<\delta$ for all $w \in U_{1}$. Define the function

$$
g(z):=\frac{1}{p(z)-p(u)} .
$$

This function belongs to $H^{\infty}\left(U_{1}\right)$, thus

$$
\widehat{C_{\varphi^{n}}}(g) \in H^{\infty}(V) .
$$

Recall that, by definition, the operator $\widehat{C_{\varphi^{n}}}$ acts as a composition operator on $\mathscr{A}(\Omega)$, but on other elements it is defined via extension from a dense subspace. Since $g$ is defined on some polynomial polyhedron containing $U_{1}$ as a relatively compact set there is a sequence $\left(g_{l}\right)_{l \in \mathbb{N}}$ of polynomials (so elements of $\mathscr{A}(\Omega)$ ) tending uniformly on $U_{1}$ to $g$. Thus

$$
\widehat{C_{\varphi^{n}}}(g)=\lim _{l \rightarrow \infty} \widehat{C_{\varphi^{n}}}\left(g_{l}\right)=\lim _{l \rightarrow \infty} g_{l} \circ \varphi^{n} .
$$

The limit is taken in $H^{\infty}(V)$, which implies that $g_{l} \circ \varphi^{n}, n \in \mathbb{N}$, are holomorphic functions defined on $V$ which uniformly tend to $\widehat{C_{\varphi^{n}}}(g)$. Without loss of generality we may assume that $K$ and $V$ are connected. Since $\varphi^{n}(K) \subseteq L \subseteq U_{1}$ we get, on a neighbourhood of $K$,

$$
g_{l} \circ \varphi^{n} \rightarrow g \circ \varphi^{n} \quad \text { as } l \rightarrow \infty,
$$

therefore $g \circ \varphi^{n}$ and $\widehat{C_{\varphi^{n}}}(g)$ coincide on some complex neighbourhood of $K$ as holomorphic functions.

Set

$$
W:=\left\{z: p \circ \varphi^{n}(z)=p(u)\right\}, \quad W \cap V \neq \emptyset
$$


The function $g \circ \varphi^{n}$ is defined on $V \backslash W$ and, since $W$ is analytic, the set $V \backslash W$ is connected. Thus on $V \backslash W$

$$
\widehat{C_{\varphi^{n}}}(g)=g \circ \varphi^{n} .
$$

Choosing $\left(v_{k}\right)_{k \in \mathbb{N}}$ in $V \backslash W$ such that $\lim _{k \rightarrow \infty} v_{k}=v \in V$ with $\varphi^{n}(v)=u$ we get

$$
\lim _{k \rightarrow \infty}\left|g \circ \varphi^{n}\left(v_{k}\right)\right|=\infty \text { and } \lim _{k \rightarrow \infty} \widehat{C_{\varphi^{n}}}(g)\left(v_{k}\right)=\widehat{C_{\varphi^{n}}}(g)(v) \text {; }
$$

a contradiction.

(d) $\Rightarrow$ (e): First we show that $U$ contains a complex neighbourhood $V$ such that $\varphi$ extends on $V$ and $\varphi(V) \subseteq V$. In case $\Omega$ is compact, this is obvious. For non-compact $\Omega$ assume that (d) holds. For any compact set $K$ we find a suitable compact $L$ and then for this $L$ we find another compact set $L_{1}$ such that for every open neighbourhood $U_{1}$ of $L_{1}$ there is a neighbourhood $U$ of $L$ such that $\varphi^{n}$ are defined on $U$ and $\varphi^{n}(U+B(0, \varepsilon)) \subseteq U_{1}$ for every $n \in \mathbb{N}$ and some fixed $\varepsilon>0$. Here $U+B(0, \varepsilon)$ means in fact the intersection of $\tilde{\Omega}$ with the algebraic sum of $U$ and $B(0, \varepsilon)$ in $\mathbb{C}^{d}$. Let us take a bounded complex neighbourhood $U_{1}$. It is easily seen that $\left(\varphi^{n}\right)$ are equicontinuous complex maps on $U$ (use Cauchy estimates). Thus

$$
\forall \delta>0 \exists 0<\eta(\delta)<\delta \forall n \in \mathbb{N}: \quad x, y \in U,\|x-y\|<\eta(\delta) \Rightarrow\left\|\varphi^{n}(x)-\varphi^{n}(y)\right\|<\delta .
$$

Let us consider a complex neighbourhood $W$ of $K, W \subseteq U$, such that for every $n \in \mathbb{N}$

$$
\varphi^{n}(W)+B(0, \varepsilon) \subseteq U .
$$

Define

$$
W_{0}:=W, \quad W_{k}:=\varphi\left(W_{k-1}\right)+B\left(0, \eta\left(\frac{\varepsilon}{2^{k}}\right)\right) .
$$

We prove inductively that

$$
\varphi^{n}\left(W_{k}\right) \subseteq\left(\bigcup_{l=1}^{\infty} \varphi^{l}(W)\right)+B\left(0, \sum_{j=1}^{k} \frac{\varepsilon}{2^{j}}\right) \quad \text { and } \quad W_{k} \subseteq U .
$$

Clearly this is true for $k=0$. Now, assuming (2) for $k-1$ we get

$$
W_{k}=\varphi\left(W_{k-1}\right)+B\left(0, \eta\left(\frac{\varepsilon}{2^{k}}\right)\right) \subseteq\left(\bigcup_{l=1}^{\infty} \varphi^{l}(W)\right)+B\left(0, \sum_{j=1}^{k-1} \frac{\varepsilon}{2^{j}}\right)+B\left(0, \eta\left(\frac{\varepsilon}{2^{k}}\right)\right) \subseteq U .
$$

Moreover, we get

$$
\begin{aligned}
\varphi^{n}\left(W_{k}\right)= & \varphi^{n}(\underbrace{\varphi\left(W_{k-1}\right)+B\left(0, \eta\left(\frac{\varepsilon}{2^{k}}\right)\right)}_{\subseteq U}) \subseteq \varphi^{n+1}\left(W_{k-1}\right)+B\left(0, \frac{\varepsilon}{2^{k}}\right) \\
& \subseteq\left(\bigcup_{l=1}^{\infty} \varphi^{l}(W)\right)+B\left(0, \sum_{j=1}^{k-1} \frac{\varepsilon}{2^{j}}\right)+B\left(0, \frac{\varepsilon}{2^{k}}\right) \subseteq\left(\bigcup_{l=1}^{\infty} \varphi^{l}(W)\right) \\
& +B\left(0, \sum_{j=1}^{k} \frac{\varepsilon}{2^{j}}\right) .
\end{aligned}
$$

Since $W_{k}$ are open, $\varphi\left(W_{k}\right) \subseteq W_{k+1}$, thus $\tilde{W}:=\bigcup_{k=0}^{\infty} W_{k}$ is an open set with $\varphi(\tilde{W}) \subseteq \tilde{W}$. 
We have proved that for every complex neighbourhood $U$ of $\Omega$ and every compact set $K \subseteq \Omega$ there is a complex open neighbourhood $W_{K}$ of $K$ such that

$$
\varphi\left(W_{K}\right) \subseteq W_{K} \subseteq U
$$

The set $V:=\bigcup_{K \Subset \Omega} W_{K}$ is a complex neighbourhood of $\Omega, \varphi(V) \subseteq V$.

Now we show that there is a fundamental sequence of compact sets $\left(K_{j}\right)$ in $\Omega$ such that $\varphi\left(K_{j}\right) \subseteq K_{j}$ for every $j \in \mathbb{N}$. Again for $\Omega$ compact this is trivial. In the general case, by (d), for every compact set $K \Subset \Omega$ there is a compact set $L \Subset \Omega$ such that $\varphi^{n}(K) \subseteq L$ for every $n \in \mathbb{N}$. Define

$$
M:=\overline{K \cup \bigcup_{n=1}^{\infty} \varphi^{n}(K)}
$$

which is a compact set and $K \subseteq M, \varphi(M) \subseteq M \subseteq L$. Therefore we can construct $\left(K_{j}\right)$ easily.

Without loss of generality we may assume that $U$ is biholomorphic to a bounded set since

$$
z \mapsto\left(\arctan z_{1}, \ldots, \arctan z_{d}\right)
$$

is a diffeomorphism of $\mathbb{R}^{d}$ onto $(-\pi / 2, \pi / 2)^{d}$ which maps biholomorphically a complex neighbourhood of the first set onto a complex neighbourhood of the second set. Thus $U$ and $V$ are hyperbolic and, by [27, Cor. 4.1.10], the Kobayashi pseudodistance $k_{V}$ on $V$ is a distance and generates its standard topology. Define

$$
U_{j}(\varepsilon):=\left\{z \in V: k_{V}\left(z, K_{j}\right)<\varepsilon\right\} .
$$

Since every holomorphic map is non-expansive with respect to the Kobayashi distance $\varphi\left(U_{j}(\varepsilon)\right) \subseteq U_{j}(\varepsilon)$. For every $j \in \mathbb{N}$ there is $\varepsilon_{j}>0$ such that $U_{j}\left(\varepsilon_{j}\right)$ is relatively compact in $W$. Then $\varphi(V) \subseteq V$ for

$$
V:=\bigcup_{j=1}^{\infty} U_{j}\left(\varepsilon_{j}\right) .
$$

Let $K$ be a compact set in $V$, then

$$
K \subset \bigcup_{j=1}^{\infty} \bigcup_{0<\varepsilon<\varepsilon_{j}} U_{j}(\varepsilon)
$$

and therefore

$$
K \subset \bigcup_{j=1}^{n} U_{j}\left(\tilde{\varepsilon}_{j}\right)
$$

for some $0<\tilde{\varepsilon}_{j}<\varepsilon_{j}$. On the other hand, $L:=\overline{\bigcup_{j=1}^{n} U_{j}\left(\tilde{\varepsilon}_{j}\right)} \supset K$ is compact and $\varphi(L) \subseteq L$.

(e) $\Rightarrow$ (f): Obvious.

(f) $\Rightarrow$ (a): Again for compact $\Omega$ this is immediate. Assume that $\Omega$ is non-compact, $\left(C_{\varphi^{n}}\right)$ are not equicontinuous and (f) holds. As usual $\Omega$ is a real analytic manifold contained in a complexification $\tilde{\Omega} \subseteq \mathbb{C}^{d}, \tilde{\Omega} \cap \mathbb{R}^{d}=\Omega$. By the Tubular Neighbourhood Theorem [34, Th. 10.19], there is a neighbourhood $T$ of $\Omega$ in $\mathbb{C}^{d}$ and a smooth retraction onto $R: T \rightarrow$ $\Omega$. Therefore there is a complex neighbourhood $V$ of $\Omega$ such that $\varphi(V) \subseteq V$ and $R(V)=\Omega$; 
in particular, all $\varphi^{n}$ are defined on $V$. In case $\Omega$ is an open set of $\mathbb{R}^{d}$ then the smooth retraction $R$ can be assumed to be the standard projection from $\mathbb{C}^{d}$ onto $\mathbb{R}^{d}$.

Proceeding with the proof, first we show the following:

Claim There is $K \Subset \Omega$ such that for every complex neighbourhood $U \subseteq V$ of $K$ we have

$$
\bigcup_{n \in \mathbb{N}} R\left(\varphi^{n}(U)\right) \text { is not relatively compact in } \Omega
$$

Obviously, $\varphi^{n}$ is well defined on $U$ for every $n \in \mathbb{N}$. Assume that the claim does not hold, i.e.,

$$
\forall K \Subset \Omega \exists U_{K} \subseteq V \text { a complex nbh. of } K: \bigcup_{n \in \mathbb{N}} R\left(\varphi^{n}\left(U_{K}\right)\right) \text { is relatively compact in } \Omega \text {. }
$$

Clearly, $\tilde{U}:=\bigcup_{K \Subset \Omega} U_{K}$ is a complex neighbourhood of $\Omega$ such that $\varphi^{n}$ are all defined on $\tilde{U}$ and for every compact subset $M$ of $\tilde{U}$ the set $\bigcup_{n \in \mathbb{N}} R\left(\varphi^{n}(M)\right)$ is relatively compact in $\Omega$.

For every complex neighbourhood $\tilde{V} \subseteq \tilde{U}$ of $\Omega$ there is another complex neighbourhood $\tilde{W}(\tilde{V}) \subseteq \tilde{V}$ such that for every $K \Subset \Omega$ the set

$$
\{z \in \tilde{W}: R(z) \in K\}
$$

is relatively compact in $\tilde{V}$. We choose $V_{0}$ a complex neighbourhood of $\Omega$ such that $V_{0} \subseteq$ $\tilde{W}(\tilde{U})$ and $\varphi\left(V_{0}\right) \subseteq V_{0}$ [it exists by (f) and the remarks above]. Analogously, we choose inductively a basis of complex neighbourhoods $\left(V_{i}\right)$ of $\Omega$ such that

$$
V_{i+1} \subseteq \tilde{W}\left(V_{i}\right), \quad \varphi\left(V_{i+1}\right) \subseteq V_{i+1}
$$

(again by (f) and the remarks above). Clearly,

$$
\varphi^{n}\left(V_{i}\right) \subseteq V_{i} \quad \forall n \in \mathbb{N}, i
$$

and since $V_{i+1} \subseteq \tilde{U}$ and $V_{i+1} \subseteq \tilde{W}\left(V_{i}\right)$ for every compact set $M \subseteq V_{i+1}$ the set $\overline{\bigcup_{n \in \mathbb{N}} \varphi^{n}(M)}$ is compact in $V_{i}$. Thus for every $f \in H\left(V_{i}\right)$, the sequence $\left(C_{\varphi^{n}}(f)\right)$ is bounded in $H\left(V_{i+1}\right)$ and thus in $\mathscr{A}(\Omega)$. We have proved that for every $f \in \mathscr{A}(\Omega)$ the sequence $\left(C_{\varphi^{n}}(f)\right)$ is bounded in $\mathscr{A}(\Omega)$ which contradicts our assumption that the sequence $\left(C_{\varphi^{n}}\right)_{n}$ is not equicontinuous on $\mathscr{A}(\Omega)$. This completes the proof of Claim.

By the claim we have just proved, $K$ has a complex neighbourhood basis $\left(U_{k}\right)$ such that for every $k$ there is a sequence of points $\left(x_{k, n}\right)_{n \in \mathbb{N}}$ in $U_{k}$ such that $R\left(\varphi^{n}\left(x_{k, n}\right)\right)$ are arbitrarily close to the boundary of $\Omega$ as $n \rightarrow \infty$. We may assume that $\varphi^{n}\left(x_{k, n}\right) \notin \mathbb{R}^{d}$, since taking $\tilde{x}_{k, n}$ close to $x_{k, n}$ we get $\varphi^{n}\left(\tilde{x}_{k, n}\right)$ close to $\varphi^{n}\left(x_{k, n}\right)$ and since $\varphi^{n}$ cannot be real on a complex open set. Let $\left(\Omega_{m}\right)_{m \in \mathbb{N}}$ be an open relatively compact exhaustion of $\Omega$. Then there is an open complex neighbourhood $W_{m}$ of $\Omega_{m}$ contained in $R^{-1}\left(\Omega_{m}\right)$ which does not contain any element of $\left\{\varphi^{n}\left(x_{k, n}\right): n \in \mathbb{N}, k \leq m\right\}$. Let $W:=\bigcup_{m \in \mathbb{N}} W_{m}$ then for any $k \in \mathbb{N}$ there are infinitely many $\varphi^{n}\left(x_{k, n}\right)$ not contained in $W$. Therefore there is no complex neighbourhood $V \subseteq W$ of $\Omega$ such that $\varphi(V) \subseteq V$. A contradiction with (f).

Remarks 1. The condition (f) of Theorem 2.1 is equivalent to the condition that for every Fréchet space $E$ continuously embedded into $\mathscr{A}(\Omega)$ there is another Fréchet space $F$ continuously embedded into $\mathscr{A}(\Omega)$ such that $C_{\varphi^{n}}(E) \subseteq F$ for every $n \in \mathbb{N}$. This is a consequence of the fact that for every Fréchet space $F$ each continuous linear map $T: F \rightarrow \mathscr{A}(\Omega)$ factorizes through some $H(U)$ for some complex neighbourhood $U$ of $\Omega$, as was proved in [15, proof of Prop. 5.2]. In fact, the Fréchet space $F$ can be chosen satisfying that $C_{\varphi^{n}}: E \rightarrow F, n \in \mathbb{N}$, form an equicontinuous sequence; see Theorem 2.1. 
2. By the result above, there is a fundamental sequence of compact subsets $\left(K_{j}\right)$ of $\Omega$ such that $\varphi\left(K_{j}\right) \subseteq K_{j}$.

Theorem 2.1 shows that power boundedness of $C_{\varphi}$ on $\mathscr{A}(\Omega)$ is equivalent to existence of many complex neighbourhoods of $\Omega$ such that $\varphi$ extends to them as a self map even with good behaviour of orbits. It turns out that it suffices to have one sufficiently small neighbourhood whenever orbits behave well.

Theorem 2.2 Let $\Omega \subseteq \mathbb{R}^{d}$ be a real analytic connected manifold and let $\varphi: \Omega \rightarrow \Omega$ be a real analytic map. Then $C_{\varphi}: \mathscr{A}(\Omega) \rightarrow \mathscr{A}(\Omega)$ is power bounded if and only if any one of the following conditions hold:

(a) There is a complex neighbourhood $V$ of $\Omega$ such that $\varphi$ extends to $V$ as a holomorphic self map and $\varphi$ has stable orbits on $V$.

(b) There is a hyperbolic complex neighbourhood $V$ of $\Omega$ such that $\varphi$ extends to $V$ as a holomorphic self-map, $V$ is contained in a Stein neighbourhood $U$ such that the inclusion $\Omega \hookrightarrow U$ is a proper map (i.e. the inverse image of every compact set is compact) and $C_{\varphi}: H(V) \rightarrow H(V)$ is power bounded.

(c) There is a hyperbolic complex neighbourhood $V$ of $\Omega$ such that $\varphi$ extends to $V$ as a holomorphic self-map and $\varphi$ has stable orbits on $\Omega$.

(d) There is a complex neighbourhood $V$ of $\Omega$ such that $\varphi$ extends to $V$ as a holomorphic self-map, $V$ is contained in a complex Kobayashi complete hyperbolic neighbourhood $U$ of $\Omega$ such that the inclusion $\Omega \hookrightarrow U$ is a proper map and $\varphi$ has at least one orbit relatively compact in $\Omega$.

First, we need a lemma.

Lemma 2.3 Every real analytic manifold has a basis of neighbourhoods in its complexification consisting of Kobayashi complete hyperbolic manifolds.

Proof By [24, Th. VI.1.1] every real analytic manifold $\Omega$ embeds into $\mathbb{R}^{d}$ as a submanifold. Taking $W=\mathbb{R}^{d} \backslash(\bar{\Omega} \backslash \Omega)$, then $\Omega$ is embedded via a proper map as a submanifold into $W$. By $[32,2.2]$, there is a real analytic map $h: W \rightarrow \mathbb{R}_{+}$such that $h(x) \rightarrow 0$ as $x \rightarrow \partial W$. Thus the map

$$
x \mapsto(x, 1 / h(x))
$$

is a proper embedding of $W$ into $\mathbb{R}^{d+1}$. Of course, there is a complexification $\tilde{W}$ contained in $\mathbb{C}^{d+1}$. On the other hand, $\mathbb{R}^{d+1}$ has a basis of Kobayashi complete hyperbolic neighbourhoods $S$ in $\mathbb{C}^{d+1}$ : just take products of Kobayashi complete hyperbolic one-connected neighbourhoods of $\mathbb{R}$ in $\mathbb{C}$, see [27, Prop. 3.2.3]. Since the complexification $\tilde{\Omega}$ of $\Omega$ in $\tilde{W}$ is closed in sufficiently small neighbourhoods $S$, the intersections $S \cap \tilde{\Omega}$ form the required basis of complex complete hyperbolic neighbourhoods of $\Omega$.

Proof of Theorem 2.2 Necessity of all conditions follows from Theorem 2.1 since every real analytic manifold has a basis of complex neighbourhoods in its complexification consisting of Stein neighbourhoods [24, Th. III.3.6] or consisting of Kobayashi complete hyperbolic neighbourhoods, see Lemma 2.3.

We show that each condition is sufficient.

Sufficiency of (a): Condition (a) implies condition (d) in Theorem 2.1 by an argument similar to the one given in the proof that (d) implies (e) in Theorem 2.1. We omit the details; compare with [9, Proposition 2.2]. 
(d) $\Rightarrow$ (a) [and (d) is also sufficient]: Let the orbit of $x \in \Omega$ be relatively compact in $\Omega$ and let $K$ be an arbitrary compact set in $\Omega$. Since $V$ is hyperbolic (as a subset of a hyperbolic set),

$$
\sup _{z \in K} k_{V}(z, x)=R<\infty .
$$

Thus for every $n \in \mathbb{N}, z \in K$, we have:

$$
k_{U}\left(\varphi^{n}(z), \varphi^{n}(x)\right) \leq k_{V}\left(\varphi^{n}(z), \varphi^{n}(x)\right) \leq k_{V}(z, x) \leq R .
$$

As the orbit of $x$ is relatively compact in $U$ we have

$$
\sup _{n \in \mathbb{N}} k_{U}\left(x, \varphi^{n}(x)\right)=C<\infty
$$

and for every $n \in \mathbb{N}$ and $z \in K$ we get

$$
k_{U}\left(\varphi^{n}(z), x\right) \leq R+C .
$$

Since $U$ is Kobayashi complete, all its balls are relatively compact in $U$, hence $\bigcup_{n \in \mathbb{N}} \varphi^{n}(K)$ is relatively compact in $U$ and contained in $\Omega$. Since $\partial_{\mathbb{R}} \Omega \subseteq \partial_{\mathbb{C}} U$, the set is also relatively compact in $\Omega$. We have proved that for every compact set $K \subseteq \Omega$ there is a compact set $L$ in $\Omega$ such that $\varphi^{n}(K) \subseteq L$ for every $n \in \mathbb{N}$. We easily construct a fundamental family $\left(K_{j}\right)$ of compact sets in $\Omega$ such that $\varphi\left(K_{j}\right) \subseteq K_{j}$ for every $j \in \mathbb{N}$.

Now, we can repeat the last part of the proof of $(\mathrm{d}) \Rightarrow$ (e) in Theorem 2.1 to get that there is complex neighbourhood $W \subseteq V$ of $\Omega$ such that there is a fundamental family of compact sets $\left(L_{j}\right)$ in $W$ with $\varphi\left(L_{j}\right) \subseteq L_{j}$ for every $j \in \mathbb{N}$. The set $W$ plays the role of $V$ in (a).

Sufficiency of (c): As in the proof of (d) $\Rightarrow$ (e) in Theorem 2.1, we find a fundamental family $\left(K_{j}\right)$ of compact sets in $\Omega$ such that $\varphi\left(K_{j}\right) \subseteq K_{j}$ for every $j \in \mathbb{N}$. For every sequence $\left(\varepsilon_{j}\right)$ of positive numbers the set

$$
W:=\left\{z \in V: \exists j \in \mathbb{N} \quad k_{V}\left(z, K_{j}\right)<\varepsilon_{j}\right\}
$$

whenever $k_{V}$ denotes the Kobayashi distance of $V$ satisfies $\varphi(W) \subseteq W$. Every neighbourhood of $\Omega$ in $V$ contains a set of the form $W$. This completes the proof by Theorem 2.1 (f).

(b) $\Rightarrow$ (c) [and (b) is also sufficient]: Assume that $C_{\varphi}: H(V) \rightarrow H(V)$ is power bounded.

For every $K$ compact in $V$ we find $L$ compact in $V$ such that $C_{\varphi^{n}}$ extends to $\widehat{C_{\varphi^{n}}}: A(L) \rightarrow$ $A(K)$ as a continuous map, where $K, L$ has non-empty interiors and $A(K)$ denotes the completion of $H(V)$ with respect to the sup-norm on $K$. Let us denote by $\hat{L}$ the holomorphic hull of $L$ in $U$, i.e.,

$$
\hat{L}=\left\{w \in U:|f(w)| \leq \sup _{z \in L}|f(z)| \forall f \in H(U)\right\} .
$$

If there is $a \in \varphi^{n}(K) \backslash \hat{L}$ then there is a function $f \in H(U) \subseteq H(V)$ such that $f(a)=1$ but $\sup _{z \in \hat{L}}|f(z)| \leq \varepsilon, \varepsilon \in(0,1)$. Since $f^{k}$ tends uniformly to zero on $\hat{L} \supseteq L$ as $k \rightarrow \infty$ but does not tend to zero at $a$, the map $C_{\varphi^{n}}$ is not continuous from $A(L)$ to $A(K)$. Since $\varphi(\Omega) \subseteq \Omega$, for every compact set $K$ in $V$ the set $\bigcup_{n=1}^{\infty} \varphi^{n}(K \cap \Omega) \subseteq \hat{L} \cap \Omega=: M$ is relatively compact in $\Omega$ (since $M$ is compact in $U$ and $\Omega$ is closed in $U$ ). Thus we have proved that for every compact set $K \subseteq \Omega$ there is a compact set $L$ in $\Omega$ such that $\varphi^{n}(K) \subseteq L$ for every $n \in \mathbb{N}$. This completes the proof of (c). 
Remark Examples of hyperbolic neighbourhoods are arbitrary complex manifolds biholomorphic with submanifolds of bounded open sets see [27, Cor. 4.1.10, Prop. 3.2.2]. For $d=1$ every domain with complement in $\mathbb{C}$ consisting of two points is hyperbolic. If $\Omega$ is a real line then $\varphi$ is real on the whole real line and then $V$ in (d) or (b) can be assumed symmetric with respect to the real line. If it has one point outside it has automatically two such points.

Problem 2.4 Is it true that power boundedness of $C_{\varphi}: \mathscr{A}(\Omega) \rightarrow \mathscr{A}(\Omega)$ implies that there exists a complete hyperbolic complex neighbourhood $V$ of $\Omega$ such that $\varphi(V) \subseteq V$ or such that this set $V$ is a domain of holomorphy? Does there exist a fundamental family of such neighbourhoods?

Slightly more can be said if the relatively compact orbit in Theorem 2.2 (d) reduces to one point. We have the following consequence of Carathéodory-Cartan-Kaup-Wu Theorem [28, 10.2.15]; see also [27, Th. 5.5.1].

Corollary 2.5 Let $\Omega$ be a real analytic connected manifold and let $\varphi: \Omega \rightarrow \Omega$ be a real analytic map with a fixed point $u \in \Omega$. If $C_{\varphi}: \mathscr{A}(\Omega) \rightarrow \mathscr{A}(\Omega)$ is power bounded then either $\left|\operatorname{det} \varphi^{\prime}(u)\right|<1$ or $\varphi$ is a biholomorphic automorphism of a (fundamental) family of hyperbolic complex neighbourhoods of $\Omega$. In the latter case, if $\varphi^{\prime}(u)$ is the identity map then $\varphi$ is the identity.

Remark Even if the first case holds orbits of $\varphi$ need not converge to a fixed point and there could exist many fixed points. Consider the map

$$
\begin{aligned}
\varphi(x, y, z):= & \left((x \cos z-y \sin z) \cdot\left(0.5+\frac{1}{2 \sqrt{x^{2}+y^{2}}}\right),\right. \\
& \left.(x \sin z+y \cos z) \cdot\left(0.5+\frac{1}{2 \sqrt{x^{2}+y^{2}}}\right), z\right)
\end{aligned}
$$

defined on a cylinder with basis in $x-y$ plane being an annulus $(z \in(-1,1))$. It is easily seen that the only fixed points are of the form $(x, y, 0)$ where $x^{2}+y^{2}=1$ but orbits starting from $(x, y, z)$ tend to the circle $\left\{(x, y, z): x^{2}+y^{2}=1\right\}$.

By Theorem 2.1 (e) and [9, Theorem 2.5] we get immediately the following consequence.

Corollary 2.6 Let $\Omega$ be a real analytic connected manifold and let $\varphi: \Omega \rightarrow \Omega$ be a real analytic map. If $C_{\varphi}: \mathscr{A}(\Omega) \rightarrow \mathscr{A}(\Omega)$ is mean ergodic then there is a real analytic submanifold $M \subseteq \Omega$ and a real analytic surjective retraction $\rho: \Omega \rightarrow M$ such that $\varphi$ restricted to $M$ is a real analytic diffeomorphism such that the smallest closed group containing $\varphi$ is a compact abelian group $G$, every $\varphi$-orbit tends to some $G$-orbit of elements of $M$ (convergence is uniform on compact sets of starting points of orbits) and

$$
P(f)(z):=\lim _{n \rightarrow \infty} \frac{1}{N} \sum_{n=1}^{N} C_{\varphi^{n}}(f)(z)=\int_{G} f(\gamma \circ \rho(z)) d H(\gamma),
$$

where $H$ is the Haar measure on $G$ and

$$
\text { im } P=\left\{f: f \text { is constant on } \rho^{-1}(\{\gamma \circ \rho(z): \gamma \in G\}) \quad z \in U\right\} .
$$

Remark By Theorem 2.2 (c), if there is a hyperbolic complex neighbourhood $V$ of $\Omega$ such that $\varphi(V) \subseteq V$ then the condition in Corollary above is also sufficient for power boundedness of $C_{\varphi}: \mathscr{A}(\Omega) \rightarrow \mathscr{A}(\Omega)$. 
Observe that all criteria for power boundedness of $C_{\varphi}: \mathscr{A}(\Omega) \rightarrow \mathscr{A}(\Omega)$ contain conditions on the behavior of $\varphi$ outside $\Omega$.

Problem 2.7 Give a characterization of power bounded composition operators $C_{\varphi}: \mathscr{A}(\Omega)$ $\rightarrow \mathscr{A}(\Omega)$ in terms of the behavior of $\varphi$ solely on $\Omega$.

In case $\Omega$ is an interval, we have always a fixed point and part (c) of the result below solves the problem above in that case.

Theorem 2.8 Let $a, b \in \overline{\mathbb{R}}$ and let $\varphi:] a, b[\longrightarrow] a, b[$ be real analytic. The following are equivalent:

(a) $C_{\varphi}: \mathscr{A}(] a, b[) \longrightarrow \mathscr{A}(] a, b[)$ is power bounded.

(b) There exists a complex neighbourhood $U$ of $] a, b[$ such that $\varphi(U) \subseteq U, \mathbb{C} \backslash U$ contains at least two points, and $\varphi$ has a (real) fixed point $u$, or equivalently, there is a fundamental family of such neighbourhoods of $] a, b[$.

(c) $\varphi$ is of one of the following forms:

(1) $\varphi=$ id ;

(2) $\varphi^{2}=\mathrm{id}$;

(3) $\varphi^{n}$ tends in $\mathscr{A}(] a, b[)$ to a constant function $\left.\equiv u \in\right] a, b\left[\right.$ as $n \rightarrow \infty$ and $\left|\varphi^{\prime}(u)\right|<1$.

If $u$ is the fixed point of $\varphi$ then the above cases in $(c)$ correspond to:

(1) $\varphi^{\prime}(u)=1$;

(2) $\varphi^{\prime}(u)=-1$;

(3) $\left|\varphi^{\prime}(u)\right|<1$.

Moreover, $C_{\varphi}$ is uniformly mean ergodic and the projection $P:=\lim _{n \rightarrow \infty} \frac{1}{N} \sum_{n=1}^{N} C_{\varphi^{n}}$ is of the following form:

(1) $P=$ id ;

(2) $P(f)=\frac{f+f \circ \varphi}{2}$, im $P=\{f: f=f \circ \varphi\}$, $\operatorname{ker} P=\{f: f=-f \circ \varphi\}$;

(3) $P(f)=f(u)$, im $P=$ the set of constant functions, ker $P=\{f: f(u)=0\}$.

Proof (a) $\Rightarrow$ (b): First, we show that there is a compact subinterval $[A, B]$ of $] a, b$ [ such that $\varphi([A, B]) \subseteq[A, B]$. Indeed, by Theorem 2.2 (c), for any compact interval $[C, D]$ of ]$a, b[$ there is a compact interval $[E, F] \subset] a, b\left[\right.$ such that $\varphi^{n}([C, D]) \subseteq[E, F]$ for every $n \in \mathbb{N}$. We define

$$
K:=\overline{[E, F] \cup \bigcup_{n=1}^{\infty} \varphi^{n}([E, F])}
$$

It is easily seen that $\varphi(K) \subseteq K$. It is connected since $\varphi^{n}([E, F])$ is connected and $[E, F] \cap$ $\varphi^{n}([E, F])$ contains $\varphi^{n}([C, D])$. Compactness of $K$ follows by the statement on $[C, D]$ applied to $[E, F]$. Thus $K=[A, B]$ for suitably chosen $A, B \in(a, b)$. Since $\varphi$ is continuous and $\varphi([A, B]) \subseteq[A, B], \varphi$ has a fixed point in $[A, B] \subseteq] a, b[$. The rest of the statement follows from Theorem 2.1 (e). See also Theorem 2.2 and the remark after its proof.

(b) $\Rightarrow$ (a): Use Theorem 2.2.

(b) $\Rightarrow$ (c): Part of (c) follows from Corollary 2.5 but we prefer to give a simple direct proof.

Let $U$ be a set satisfying (b) (without loss of generality we may assume that $U$ is connected), contained in $\{z \in \mathbb{C}: d(z] a,, b[)<1, \operatorname{Re}(z) \in] a, b[\}$. Since $\varphi$ is real on the real 
line $\varphi(\bar{z})=\overline{\varphi(z)}$ for $z \in U$ and thus we may assume that $U$ is symmetric with respect to the real line. Let $\tilde{U}$ be the union of $U$ and all its compact components of the complement. Therefore there is a Riemann (biholomorphic) map $\psi: \tilde{U} \rightarrow \mathbb{D}, \mathbb{D}$ the unit disc, such that $\psi(] a, b[)=]-1,1[, \psi(u)=0, u$ the fixed point of $\varphi$ and let $W:=\psi(U)$. The maps $C_{\psi^{-1}}: H(U) \rightarrow H(W)$ and $C_{\psi}: H(W) \rightarrow H(U)$ are isomorphisms and under these isomorphisms the map $C_{\varphi}$ corresponds to $C_{\eta}$, where $\eta:=\psi \circ \varphi \circ \psi^{-1}$.

Summarizing, we can assume that $U$ is bounded, contained in the unit disc and $u=0$.

Define

$$
g_{n}(z):= \begin{cases}\frac{\varphi^{n}(z)}{z} & \text { if } z \neq 0 \\ \left(\varphi^{n}\right)^{\prime}(0) & \text { if } z=0 .\end{cases}
$$

These functions are well defined on $U$ and by the maximum principle and the Cauchy estimate $\left|g_{n}(z)\right| \leq 1 / \operatorname{dist}(0, \partial U)=$ : $C$. Hence $\left|\left(\varphi^{n}\right)^{\prime}(0)\right| \leq C$ but $\left(\varphi^{n}\right)^{\prime}(0)=\left(\varphi^{\prime}(0)\right)^{n}$ and we get $\left|\varphi^{\prime}(0)\right| \leq 1$.

Since $\varphi$ is real on the real line $\varphi^{\prime}(0)$ must be real. If $\varphi^{\prime}(0)=1$ then, by [28, Prop. 10.1.1], $\varphi(z) \equiv z$. If $\varphi^{\prime}(0)=-1$ then $C_{\varphi^{2}}$ satisfies the same assumptions on $U$ as $C_{\varphi}$ but $\left(\varphi^{2}\right)^{\prime}(0)=1$. Thus $\varphi^{2}=$ id. If $\left|\varphi^{\prime}(0)\right|<1$ then $u$ is an attracting fixed point thus, by [36, Th. 5.2], $\varphi^{n}$ tends uniformly on compact subsets of $U$ to $u$.

By Theorem 2.1, $C_{\varphi}$ is mean ergodic and $\frac{1}{N} \sum_{n=1}^{N} C_{\varphi^{n}} \longrightarrow P$, where $P$ is a projection onto ker (id $-C_{\varphi}$ ) with kernel $\overline{\mathrm{im}\left(\mathrm{id}-C_{\varphi}\right)}$. Calculating $P$ is easy on the basis of the above data.

(c) $\Rightarrow$ (a): Cases (1) and (2) are obvious. In case (3) there is a neighbourhood $A$ of $u$ where $\varphi$ is moving all points towards $u$. For every compact subset of $] a, b$ [ there is a complex neighbourhood $B$ of it mapped by some $\varphi^{n}$ into $A$. The result follows by Theorem 2.1 (d).

Remarks 1. The equivalences of (b) and (c) with (a) in Theorem 2.8 do not hold for $d>1$. For instance take $\Omega$ an annulus in $\mathbb{R}^{2}$ and $\varphi$ a rotation.

2. If $U=\mathbb{C}$ then the condition (b) is not sufficient for (a) in Theorem 2.8 , since $\varphi(z):=2 z$ has zero as a fixed point but there are plenty of unbounded orbits.

3. In the case of several variables, power boundedness of $C_{\varphi}$ does not imply the existence of a fixed point for $\varphi$.

Example 2.9 Even if $\varphi:]-1,1[\rightarrow]-1,1$ [ maps all bounded sets in ] - 1, 1[ in one compact set it does not follow that $\varphi$ is power bounded.

Indeed, take

$$
\varphi_{\alpha}(z):=\frac{2 i}{\alpha \cdot \pi} \ln \left(\frac{1-i z}{1+i z}\right) .
$$

Clearly, $\varphi_{\alpha}$ maps the unit disc onto the vertical strip with $\operatorname{Re} z \in\left(-\frac{1}{\alpha}, \frac{1}{\alpha}\right)$. Moreover $\varphi_{\alpha}(i)=\infty$, and $\varphi_{\alpha}$ maps the real line into the real line and the imaginary line into the imaginary line. The imaginary part of converse map $\varphi_{\alpha}^{-1}(i t)$ treated as the function of the real variable $t \in$ ] $-1,1$ [ has a derivative with maximum at zero equal to $\frac{\pi \cdot \alpha}{4}$ thus if the latter is $<1$ the zero point is an attractive fixed point on the imaginary line for $\varphi_{\alpha}^{-1}$. This implies that $\varphi_{\alpha}^{-n}(i) \rightarrow 0$ as $n \rightarrow \infty$. Since on this sequence $\varphi_{\alpha}^{n+1}$ is not defined there is no common neighbourhood of zero such that all $\varphi_{\alpha}^{n}$ are defined for all $n \in \mathbb{N}$ and fixed $\alpha, \alpha<1, \frac{\pi \cdot \alpha}{4}<1$. Thus $C_{\varphi}: \mathscr{A}(]-1,1[) \rightarrow \mathscr{A}(]-1,1[)$ is not power bounded. 
Here is another example: $\varphi:]-1,1[\rightarrow]-1,1\left[, \varphi(x)=\frac{x}{1+x^{2}}\right.$. By Theorem $2.8, C_{\varphi}$ is not power bounded since $\varphi^{\prime}(0)=1$ and $\varphi^{n} \rightarrow 0$. Observe that $\left.\varphi(]-1,1[)=\right]-1 / 2,1 / 2[$. By Theorem 2.2, there is no hyperbolic complex neighbourhood $V$ such that $\varphi(V) \subseteq V$. Of course, since $\varphi$ has two singularities $i$ and $-i$ so there is no complex neighbourhood $V$ of ] $-1,1$ [ at all such that $\varphi$ is a self-map of $V$.

For purposes of comparison with our results above, we conclude here with the following result about hypercyclic composition operators on spaces of real analytic functions. Part (2) below is a consequence of our Main Theorem. Part (1) is elementary.

Corollary 2.10 Let $\Omega$ be a real analytic connected manifold and let $\varphi: \Omega \rightarrow \Omega$ be a real analytic map. If $C_{\varphi}$ is hypercyclic, then

(1) $\varphi$ is injective, its derivative $\varphi^{\prime}(z)$ at arbitrary point $z \in \Omega$ is never a singular linear map and $\varphi$ runs away, i.e., for every compact set $K \subseteq \Omega$ there is $n \in \mathbb{N}$ such that $\varphi^{n}(K) \cap K=\emptyset$.

(2) There is a complex neighbourhood $U$ of $\Omega$ such that for every complex neighbourhood $V \subseteq U$ of $\Omega$ the map $\varphi$ does not extend as a holomorphic self map on $V$.

Proof (1) If $\varphi(z)=\varphi(w)$ then every function in the image of $C_{\varphi^{n}}$ has the same values in $z$ and in $w$, thus $C_{\varphi^{n}}$ cannot be hypercyclic. If $\varphi^{\prime}(z) v=0$ for a tangent vector $v$, then inductively $\left(\varphi^{n}\right)^{\prime}(z) v=0$ for every $n \in \mathbb{N}$ and every function in im $C_{\varphi^{n}}$ has a derivative at $z$ vanishing on the vector $v$. Again $C_{\varphi}$ cannot be hypercylic. If $\varphi^{n}(K) \cap K \neq \emptyset$ for every $n \in \mathbb{N}$ then for any function $f \in \mathscr{A}(\Omega)$ with $f(K) \subseteq B(0, r)$ the function $C_{\varphi^{n}}(f)$ takes some values in $B(0, r)$ and so it cannot approximate any function with all values bigger than $r$.

Parts (2) follows form our Main Theorem.

Acknowledgments The research of Bonet was partially supported by MEC and FEDER Project MTM200762643 and by GV Project Prometeo/2008/101. The research of Domański was supported in years 2007-2010 by Ministry of Science and Higher Education, Poland, Grant no. NN201 2740 33. The authors are very indebted to F. Bracci for providing some information concerning complex dynamics. The second named author is very grateful to colleagues from Valencia for warm hospitality during his stays there.

Open Access This article is distributed under the terms of the Creative Commons Attribution Noncommercial License which permits any noncommercial use, distribution, and reproduction in any medium, provided the original author(s) and source are credited.

\section{References}

1. Abate, M.: Iteration Theory of Holomorphic Maps on Taut Manifolds. Mediterranean Press, Commenda di Rende (1989)

2. Albanese, A.A., Bonet, J., Ricker, W.J.: On mean ergodic operators. In: Curbera, G.P. et. al. (eds.) Vector Measures, Integration and Related Topics. Operator Theory: Advances and Applications, vol. 201, pp. 1-20. Birkhäuser Verlag, Basel (2009)

3. Albanese, A.A., Bonet, J., Ricker, W.J.: Mean ergodic operators in Fréchet spaces. Anal. Acad. Sci. Fenn. Math. 34, 401-436 (2009)

4. Bayart, F., Matheron, E.: Dynamics of linear operators. Cambridge Tracts in Mathematics, vol. 179. Cambridge University Press, Cambridge (2009)

5. Bernal-González, L., Montes-Rodríguez, A.: Universal functions for composition operators. Complex Variables 27, 47-56 (1995)

6. Bernal-González, L.: Universal entire functions for affine endomorphisms of $\mathbb{C}^{\mathbb{N}}$. J. Math. Anal. Appl. 305, 690-697 (2005)

7. Bonet, J., Domański, P.: Real analytic curves in Fréchet spaces and their duals. Mh. Math. 126, 13-36 (1998) 
8. Bonet, J., Domański, P.: The splitting of exact sequences of PLS-spaces and smooth dependence of solutions of linear partial differential equations. Adv. Math. 217, 561-585 (2008)

9. Bonet, J., Domański, P.: A note on mean ergodic composition operators on spaces of holomorphic functions. Rev. R. Acad. Cien. Ser. A Mat. RACSAM (to appear)

10. Cascales, B., Ka̧kol, J., Saxon, S.A.: Metrizability vs. Fréchet-Urysohn property. Proc. Am. Math. Soc. 131, 3623-3631 (2003)

11. Cowen, C.C., MacCluer, B.D.: Composition operators on spaces of analytic functions. In: Studies in Advanced Mathematics. CRC Press, Boca Raton (1995)

12. Domański, P.: Classical PLS-spaces: spaces of distributions, real analytic functions and their relatives. In: Ciesielski, Z., Pełczyński, A., Skrzypczak, L. (eds.) Orlicz Centenary Volume, vol. 64, pp. 51-70. Banach Center Publications, Institute of Mathematics, Warszawa (2004)

13. Domański, P.: Real analytic parameter dependence of solutions of differential equations. Rev. Mat. Iberoamericana 26, 175-238 (2010)

14. Domański, P.: Notes on real analytic functions and classical operators. Lecture Notes for Winter School in Complex Analysis and Operator Theory, Valencia (preprint, 2010). http://amu.edu.pl/ domanski/ valenciawinter10/Articlemode.pdf

15. Domański, P., Langenbruch, M.: Composition operators on spaces of real analytic functions. Math. Nach. 254(255), 68-86 (2003)

16. Domański, P., Langenbruch, M.: Coherent analytic sets and composition of real analytic functions. J. Reine Angew. Math. 582, 41-59 (2005)

17. Domański, P., Langenbruch, M.: Composition operators with closed image on spaces of real analytic functions. Bull. Lond. Math. Soc. 38, 636-646 (2006)

18. Domański, P., Vogt, D.: The space of real analytic functions has no basis. Stud. Math. 142, 187-200 (2000)

19. Domański, P., Vogt, D.: Linear topological properties of the space of real analytic functions on the real line. In: Bierstedt, K.D., Bonet, J., Maestre, M., Schmets, J., (eds.) Recent Progress in Functional Analysis, pp. 113-132. Proc. Valencia 2000, North-Holland Mathematical Studies, Amsterdam (2001)

20. Fonf, V.P., Lin, M., Wojtaszczyk, P.: Ergodic characterizations of reflexivity in Banach spaces. J. Funct. Anal. 187, 146-162 (2001)

21. Grosse-Erdmann, K.G.: Universal families and hypercyclic operators. Bull. Am. Math. Soc. 36, 345381 (1999)

22. Grosse-Erdmann, K.G., Mortini, R.: Universal functions for composition operators with non-automorphic symbol. J. d'Analyse Math. 107, 355-376 (2009)

23. Grosse-Erdmann, K.G., Peris, A.: Linear Chaos. Springer, Berlin (to appear, 2010)

24. Guaraldo, F., Macrì, P., Tancredi, A.: Topics on Real Analytic Spaces. Vieweg, Braunschweig (1986)

25. Gunning, R.C.: Introduction to Holomorphic Functions of Several Variables. Wadsworth \& BrooksCole, Belmont (1990)

26. Hörmander, L.: An Introduction to Complex Analysis in Several Variables, 3rd edn. Elsevier, NorthHolland, Amsterdam (1990)

27. Kobayashi, S.: Hyperbolic Complex Spaces. Springer, Berlin (1998)

28. Krantz, S.: Function Theory of Several Complex Variables. Wiley, New York (1982)

29. Krengel, U.: Ergodic Theorems. de Gruyter, Berlin (1985)

30. Kriegl, A., Michor, P.W.: The convenient setting for real analytic mappings. Acta Math. 165, 105159 (1990)

31. Kriegl, A., Michor, P.W.: The Convenient Setting of Global Analysis. American Mathematical Society, Providence (1997)

32. Langenbruch, M.: Analytic extension of smooth functions. Results Math. 36, 281-296 (1999)

33. Langenbruch, M.: Characterization of surjective partial differential operators on spaces of real analytic functions. Stud. Math. 162, 53-96 (2004)

34. Lee, J.M.: Introduction to Smooth Manifolds. Springer, New York (2003)

35. Meise, R., Vogt, D.: Introduction to Functional Analysis. Clarendon Press, Oxford (1997)

36. Milnor, J.: Dynamics in One Complex Variable. Vieweg, Braunschweig (2006)

37. Shapiro, J.H.: Composition Operators and Classical Function Theory, Universitext: Tracts in Mathematics. Springer, New York (1993)

38. Shapiro, J.H.: Notes on the dynamics of linear operators. Lecture notes. http://www.mth.msu.edu/ shapiro/Pubvit/Downloads/LinDynamics/LynDynamics.html

39. Vogt, D.: Extension operators for real analytic functions on compact subvarieties of $\mathbb{R}^{d}$. J. Reine Angew. Math. 606, 217-233 (2007)

40. Vogt, D.: Section spaces of real analytic vector bundles and a theorem of Grothendieck and Poly. In: Kaminski, A., Oberguggenberger, M., Pilipovic, S. (eds.) Generalized Functions and its Applications, vol. 88, pp. 315-321. Banach Center Publication, Warszawa (2010)

41. Yosida, K.: Functional Analysis. Springer, Heidelberg (1965) 